\title{
Supramolecular Structure of a New Family of Circular Proteoglycans Mediating Cell Adhesion in Sponges
}

\author{
J anina J archow,* J ürgen Fritz,† Dario Anselmetti,‡ Anthony Calabro,§ Vincent C. Hascall,§ \\ Daniela Gerosa,* Max M. Burger,* and Xavier Fernàndez-Busquets*,ף,1
}

\begin{abstract}
*Friedrich Miescher-Institut, CH-4002 Basel, Switzerland; †MIT Media Laboratory, 20 Ames Street, Cambridge, Massachusetts 02139; ‡Department of Experimental Biophysics, University of Bielefeld, Universitätsstrasse 25, D-33501 Bielefeld, Germany; $\S D e p a r t m e n t$ of Biomedical Engineering, Lerner Research Institute, The Cleveland Clinic Foundation, 9500 Euclid Avenue, Cleveland, Ohio 44195; and "Departament de Bioquímica i Biologia Molecular, Facultat de Farmàcia, Universitat de Barcelona, Avinguda Diagonal 643, E-08028 Barcelona, Spain
\end{abstract}

Received April 19, 2000, and in revised form J uly 21, 2000; published online December 7, 2000

\begin{abstract}
Aggregation factors are the molecules responsible for species-specific cell adhesion in sponges. Here, we present the structure of the aggregation factor from the marine sponge Microciona prolifera, which constitutes the first description of a circular proteoglycan. We have analyzed chemically dissociated and enzymatically digested aggregation factor with atomic force microscopy, agarose gel electrophoresis, and Western blots using antibodies against the protein and carbohydrate moieties. Twenty units from each of two $\mathrm{N}$-glycosylated proteins, MAF p3 and MAF p4, form the central ring and radiating arms, respectively, stabilized by a hyaluronidase-sensitive component. MAFp3 carries a 200-kDa glycan involved in homologous self-interactions between aggregation factor molecules, whereas MAFp4 carries a 6-kDa glycan that binds cell surface receptors. A 68-kDa lectin found in cell membranes of several sponge species binds the aggregation factor and its protein-free glycans, as well as chondroitin sulfate and hyaluronan. Here, we show that despite their lack of clear sequence homologies with other known proteoglycan structures, the protein and carbohydrate components of sponge aggregation factors assemble to form a supramolecular complex remarkably similar to classical proteoglycans. ๑ 2000 Academic Press

Key Words: atomic force microscope; cell adhesion; hyaluronidase; Porifera; proteoglycan.
\end{abstract}

\section{INTRODUCTION}

Proteoglycans mediate specific matrix interactions and biological activities related to different

\footnotetext{
1 To whom correspondence should be addressed. Fax: 34-934021896. E-mail: xavi@farmacia.far.ub.es.
}

aspects of cell adhesion (reviewed in Hardingham and Fosang, 1992; I ozzo and Murdoch, 1996; I Ozzo, 1998), but many of their roles in these cellular processes are still poorly understood. The mechanism for species-specific cell adhesion in sponges consists of two components present on all cells, namely, the extracellular aggregation factor (AF) and a cell membrane receptor (Weinbaum and Burger, 1973). Based on their composition and on their electron microscope images, sponge AFs were described as proteoglycan-like molecules showing either a linear appearance or a sunburst-like morphology (Figs. 1A and $1 B$ ). The purification of proteoglycans is often complicated by (i) limited source quantities, (ii) the requirement of chaotropic solvents for efficient extraction, and (iii) the lack of defined assessments of purity (F edarko, 1993). In this respect, sponge AFs could be one of the best potential models to study proteoglycan structure and function, given (i) their extraordinary abundance in the sponge extracellular matrix, (ii) the ease of their extraction in large quantities simply by removal of extracellular $\mathrm{Ca}^{2+}$, and (iii) the availability of a quick and reliable aggregation assay to quantify their activity. However, the inclusion of AFs in the proteoglycan or proteoglycan-like family awaits a precise description of how the different glycan and protein components are distributed.

Remarkably, when dissociated sponge cells from two different species are mixed together, the mixed aggregates initially formed gradually sort out according to their species of origin, in the same way as mixtures of dissociated embryonic cells from two vertebrate tissues sort out according to their tissue of origin. The molecular basis of this selective cellcell adhesion in most multicellular animals is medi- 
ated by two distinct classes of molecules: a $\mathrm{Ca}^{2+}$ independent activity like that typical of the glycoproteins from the I g superfamily (Edelman and Crossin, 1991) and a $\mathrm{Ca}^{2+}$-dependent cell-cell adhesion whose best example is the cadherins (Takeichi, 1988). Interestingly, AFs reunite in the same molecule both a $\mathrm{Ca}^{2+}$-dependent AF-AF homophilic binding and $\mathrm{C} \mathrm{Ca}^{2+}$-independent heterophilic interaction of AF with the cell receptor (J umblatt et al., 1980). At present the AF from the red beard sponge, Microciona prolifera, is best characterized. Carbohydrate structures in the Microciona AF (MAF) mediate species-specific adhesion of sponge cells (Fig. 1C), via a bifunctional activity that involves (i) a heterologous interaction with cell membrane receptors through a small glycan with an apparent mass of $6.3 \mathrm{kDa}$, termed $\mathrm{g}-6$, that is released from MAF upon peptide-N-glycosidase (PNGase) F treatment (Misevic and Burger, 1990) and (ii) homologous $\mathrm{Ca}^{2+}$-dependent interactions of a larger MAF glycan with an apparent mass of $\sim 200$ kDa termed g-200 (Misevic and Burger, 1993).

The binding of AFs to sponge cells triggers a wide variety of cellular responses. The addition of purified $A F$ to primary cell aggregates of the marine sponge Geodia cydonium resulted in increased DNA, RNA, and protein synthesis, as well as in a higher mitotic activity (Müller et al., 1976, 1994; Pfeifer et al., 1993). Binding of AF to the cells also triggered protein phosphorylation (Rottmann et al., 1987) and ras gene expression (Schröder et al., 1988). The main proteins of MAF, MAFp3 (ranging from 30 to 50 $\mathrm{kDa}$ ) and MAFp4 ( $400 \mathrm{kDa})$, are extremely polymorphic molecules (Fernàndez-Busquets and Burger, 1997). Restriction fragment length polymorphism analysis of a sponge population revealed that any two individuals whose grafted tissues were mutually rejected exhibited different band patterns using MAFp3 and MAFp4 DNA probes, suggesting an involvement of MAF-related proteins in sponge histocompatibility (Fernàndez-Busquets et al., 1998).

The extracellular matrix of sponges is very similar to that of all Metazoans (Fernàndez-Busquets and Burger, 1999), thus suggesting that the study of molecules from sponge extracellular matrices can be a good model to better understand the functions of their vertebrate counterparts. In this work we show that although MAFp3, MAFp4, g-6, and g-200 are not directly related to any other described proteoglycan components, the structure of MAF has characteristics that resemble the aggregates of cartilage proteoglycans. Unlike any proteoglycan described to date, however, the central backbone is circularized.

\section{MATERIALS AND METHODS}

Sponges and preparation of AFs, glycans, cells, and cell membranes. Live specimens of M. prolifera and Halichondria panicea were collected by the supply department of the Marine Biological Laboratory (MBL; Woods Hole, MA). Live specimens of Suberites (Ficulina) ficus were collected by the Observatoire Océanologique, Roscoff, France. Sponges were kept in running seawater and used within 3 days after collection. $\mathrm{Ca}^{2+}$ - and $\mathrm{Mg}^{2+}$-free seawater was prepared according to the MBL formula and buffered with 20 mM Tris, pH 7.4 (CMFTSW). CaCMFTSW is CMFTSW containing $2 \mathrm{mM} \mathrm{CaCl}$. AFs were isolated and purified as described (Misevic et al., 1987). After dialysis of the AFs against CaCMFTSW, protein concentrations were determined using the Bio-Rad DC Protein Assay, and the cell aggregation activities were measured in serial dilutions as described (J umblatt et al., 1980; Henkart et al., 1973). AFs were adjusted to a final concentration of $1 \mathrm{mg}$ protein $/ \mathrm{ml}$ and stored at $4^{\circ} \mathrm{C}$ in CaCMFTSW containing $0.05 \% \mathrm{NaN}_{3}$. Glycans were prepared from delipidated MAF samples by extensive pronase digestion as described (Misevic et al., 1987; Finne and Krusius, 1982). Fractions were checked for the presence of acidic glycans by dotting aliquots on a Zeta-Probe membrane (Bio-Rad), followed by staining with Alcian blue $\left(0.5 \% \mathrm{w} / \mathrm{v}\right.$ in $\left.\mathrm{H}_{2} \mathrm{O}\right)$. Suspensions of chemically dissociated sponge cells were obtained as described ( $\mathrm{J}$ umblatt et al., 1980). Cell membranes were prepared as previously reported (Fernàndez-Busquets et al., 1998).

AFs: Dissociation, chemical deglycosylation, and enzymatic treatments. EDTA dissociation and purification of the AF fragments were done as described (Humphreys et al., 1977). Dissociative density gradients in the presence of $4 \mathrm{M}$ guanidine hydrochloride $(\mathrm{GuHCl})$ were performed as described (FernàndezBusquets et al., 1996). Trifluoromethanesulfonic acid (TFMS) deglycosylation was done according to Edge et al. (1981) as described (Fernàndez-Busquets et al., 1996). In general, 12 units of Streptomyces hyalurolyticus hyaluronidase (Merck) was used to digest samples containing $\sim 30 \mu \mathrm{g}$ of carbohydrate in $10 \mathrm{mM}$ citrate, $\mathrm{pH} 5$, with overnight incubation at $37^{\circ} \mathrm{C}$. PNGase F digestions were done as specified (Fernàndez-Busquets and Burger, 1997).

Biotinylation of AFs and glycans, electrophoretic analyses, and blots. AFs (1 mg of protein/ml) were biotinylated with biotin-6aminocaproic acid-hydroxysuccinimidester (Fluka) as described (J archow and Burger, 1998). Glycans obtained after pronase digestion were labeled under the same conditions, except that a large molar excess of the reactive agent (at least 20 -fold) was added for amino-terminal labeling of the remaining amino acid residue. Labeled glycans were separated from free reagents by elution on a G-25 sizing column (Amersham Pharmacia Biotech) in $100 \mathrm{mM}$ pyridine-acetate, $\mathrm{pH}$ 5.0, and the glycan fraction was subsequently lyophilized. SDS-polyacrylamide gel electrophoresis (PAGE) was done as described by Laemmli (1970). Western blots were done as described (Fernàndez-Busquets et al., 1996). Binding of biotinylated AFs or glycans to blotted molecules was done in blocking buffer ( $3 \%$ bovine serum albumin (BSA) in CaCMFTSW) for either $2 \mathrm{~h}$ at room temperature or overnight at $4^{\circ} \mathrm{C}$ with final concentrations from 5 to $30 \mu \mathrm{g} / \mathrm{ml}$ for AFs and 50 to $200 \mu \mathrm{g} / \mathrm{ml}$ for glycans. Blots were washed twice in blocking buffer and bound AFs were detected by incubating with extravidin-horseradish peroxidase in blocking buffer for $1 \mathrm{~h}$ at room temperature. The enhanced chemiluminiscence Western blotting detection system (Amersham Pharmacia Biotech) was used to visualize the decorated bands. Tris- borate-EDTA (TBE)-PAGE of glycans was done in $15 \%$ gels essentially as described (Misevic, 1989), but modified for the Bio-Rad minigel system. The running buffer was precooled to $4^{\circ} \mathrm{C}$ and electrophoresis was carried out at 
200 V. Gels were stained with Alcian blue. Agarose gel electrophoresis was done in $100 \mathrm{mM}$ Tris, $100 \mathrm{mM}$ Tricine, $15 \mathrm{mM}$ $\mathrm{CaCl}_{2}$. Electrophoresis was done at $30 \mathrm{~V}$ for 6 to $8 \mathrm{~h}$ and the gels were subsequently stained for acidic glycans with toluidine blue (Björnsson, 1993). MAF subunits electrophoresed in $0.5 \times$ TBE $-1 \%$ agarose gels at $40 \mathrm{~V}$ were transferred to a positively charged nylon membrane (Boehringer Mannheim) by capillary transfer as described for nucleic acids (Sambrook et al., 1989). Membranes were blocked in Tris-buffered saline, pH 7.5, 3\% BSA, $0.5 \%$ Tween $20,1 \%$ Triton X-100 for $1 \mathrm{~h}$. Incubation with the first antibody was done at room temperature for $2 \mathrm{~h}$ or overnight at $4^{\circ} \mathrm{C}$ using 2-5 $\mu \mathrm{g}$ of antibody/ml of blocking solution. After being washed, the blots were incubated with the corresponding horseradish peroxidase-coupled secondary antibody in blocking solution at room temperature for $1 \mathrm{~h}$. Detection of the decorated bands was done as described above. Fluorophore-assisted carbohydrate el ectrophoresis (FACE) was done according to Calabro et al. (2000). Generally, $25 \mu \mathrm{g}$ of streptococcal or S. hyalurolyticus hyaluronidase-digested aggregation factor samples was analyzed. Controls done by spiking known amounts of hyaluronan and chondroitin sulfate determined that $\geq 25 \mathrm{ng}$ of hyaluronan in the original sample would be detected.

Bead coupling, aggregation assays, and microscopy. Coupling of AF fractions to amine beads (Molecular Probes, diameter $1 \mu \mathrm{m}$ ) was done by incubating the beads for $2 \mathrm{~h}$ at room temperature in PBS containing $8 \%$ glutaral dehyde. Beads were then washed up to 10 times with PBS to remove unbound glutaraldehyde. AF fractions were added in large excess, and coupling was allowed to proceed overnight at $37^{\circ} \mathrm{C}$. Remaining free glutaral dehyde groups were then quenched by incubation in $100 \mathrm{mM}$ Tris, $100 \mathrm{mM}$ Tricine, $\mathrm{pH} 8.3$, for $2 \mathrm{~h}$, and beads were washed 5 times with the same buffer. Coupling efficiency was determined by dotting a bead aliquot on a Zeta-Probe membrane followed by staining with Alcian blue. Bead aggregation assays were done on a rotary shaker in wells constructed for microscopic examination by mounting a plastic ring onto a coverslip. Two to $10 \mu \mathrm{l}$ of beads was suspended in $400 \mu \mathrm{l}$ of CMFTSW, and $\mathrm{Ca}^{2+}$ was added to a final concentration of $10 \mathrm{mM}$. I mages of bead aggregates were acquired with a confocal laser scanning microscope (Leica Lasertechnik, Heidelberg, Germany) equipped with an Ar/Kr laser and a 10X objective. The size of the scanned area was $1 \times 1 \mathrm{~mm}$ and digitized images consisted of $512 \times 512$ pixels. Image processing was done using a commercial software product (I maris by Bitplane, Zürich, Switzerland). Cell aggregation assays were done in 24-well plates with $2 \times 10^{7}$ cells/ml in a final volume of $275 \mu \mathrm{l}$ of CaCMFTSW. Atomic force microscope (AFM) experiments were done with the commercial instruments Nanoscope III and IIIa (Multimode and Bioscope AFM, Digital Instruments, Santa Barbara, CA). I maging was done in tapping mode of operation under ambient conditions with standard Si cantilevers (Nanoprobe, Wetzlar, Germany). Sample preparation was al ways according to our standard protocols (Fritz et al., 1997) or according to the protocols using $\mathrm{NH}_{2}$-functionalized mica substrates (Lyubchenko et al., 1993).

Affinity columns and antibodies. Coupling of either MAF or glycosaminoglycans to EAH-Sepharose (Amersham Pharmacia Biotech) was done as suggested by the manufacturer. Binding of crude membrane extracts to affinity columns was done in CaCMFTSW at $2 \mathrm{mM} \mathrm{Ca}^{2+}$ for $20 \mathrm{~min}$ on ice. Small column batches were then washed twice in CaCMFTSW and once in distilled water to reduce salt concentrations prior to boiling in sample buffer containing $2 \%$ SDS. The monoclonal antibody Block 2 was purified from cellular supernatants by ammonium sulfate precipitation and further via a Protein A column. Polyclonal rabbit I gGs against MAF p3 and MAF p4 were produced and purified according to standard protocols (Harlow and Lane, 1988). Fusion proteins were generated with the Glutathione S-Transferase Gene F usion System (Amersham Pharmacia Biotech). The MAF p3 fusion protein contained the recombinant 170-amino-acid amino-terminal region of MAFp3 against which chicken polyclonal antibodies had al ready been made (Fernàndez-Busquets et al., 1998). The MAFp4 fusion protein included the 353 residues corresponding to domains 14, 15, and 16 in MAF p4C (FernàndezBusquets et al., 1998). The specific antibodies against MAF p3 and MAFp4 were purified with antigen affinity columns as described (Fernàndez-Busquets et al., 1998). The eluted preparation was monitored for purity in SDS-PAGE and for its antigen-binding activity, and finally it was quantitated. As a last step each affinity column-purified antibody solution was further purified by the immunoblotting technique (Harlow and Lane, 1988), using blots where TFMS-deglycosylated MAFp3 and MAFp4 had been previously transferred. Fab fragments of the purified antibodies were generated as described (Harlow and Lane, 1988) and finally separated from Fc fragments through a Protein A column.

\section{RESULTS}

\section{EDTA Dissociation of MAF}

EDTA-treated MAF separates on a sizing column into (i) a protein- and carbohydrate-containing peak eluting in the excluded volume of the column that accounts for $\sim 30 \%$ of the starting material and (ii) a protein- and carbohydrate-containing peak of small fragments. AFM visualization of both fractions showed that the excluded volume contained the ring of the MAF molecules composed of bead-like structures (Fig. 2B). Often, rings were observed to form dimers and larger groupings. In images of native MAF the average length of an arm is $143 \pm 5 \mathrm{~nm}$, and its height is $1.2 \pm 0.1 \mathrm{~nm}$ (Fig. 2A, arrows). Linear structures with a height of $0.6 \pm 0.1 \mathrm{~nm}$ are also observed (Fig. 2A, arrowheads). These are also present in the excluded fraction ( $\mathrm{F}$ igs. 2B and $2 \mathrm{C}$ ), from where arms are completely absent. These linear structures are thinner than the arms and are twice as long $(287 \pm 7.5 \mathrm{~nm})$. This length coincides with the average circumference of a ring $(283 \pm 7.8$ $\mathrm{nm})$, suggesting that both structures might be aligned side by side in the native structure. The relative stoichiometry of rings and these rod-like molecules is $\sim 1: 1$. The height of the bead-like structures in the ring is $2.0 \pm 0.3 \mathrm{~nm}$. Table I gives the molecular dimensions of the AFs from three different species as estimated from AFM measurements. AFM images of the included fraction do not reveal any arm-like structures (data not shown), suggesting that some degradation of the arm structure occurred during the incubation in the presence of EDTA.

When coupled to synthetic beads, only fractions from the excluded volume showed $\mathrm{Ca}^{2+}$-dependent aggregation (Fig. 3A). At a $\mathrm{Ca}^{2+}$ concentration of 15 $\mathrm{mM}$, intact MAF retains the excluded fraction but 

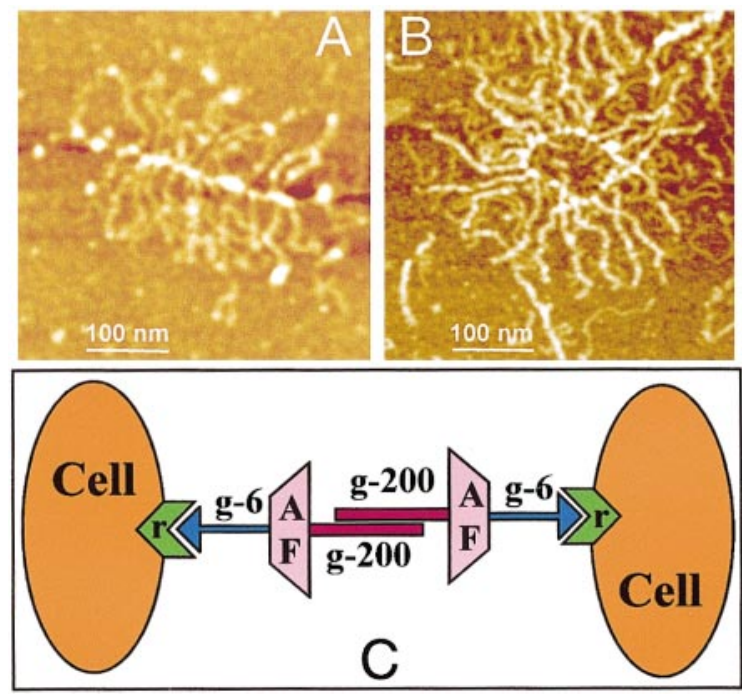

FIG. 1. AFs mediate specific sponge cell adhesion. (A) AFM image of Halichondria panicea $A F(H A F)$. (B) AFM image of Microciona prolifera AF. The color-encoded vertical z-scale of both AFM images corresponds to $3 \mathrm{~nm}$. (C) components involved in AF-mediated cell adhesion in the marine sponge $M$. prolifera: g-200, glycan mediating homologous AF-AF interactions; g-6, glycan binding the cell surface receptor ( $r$ ); AF, core protein.

not the included fraction, whereas AF from another species retains neither fraction (Fig. 3C). MAF glycans from both fractions were identified by Alcian blue staining after pronase digestion and separation by TBE-PAGE. The excluded peak contained the g-200 glycan, whereas the included fraction contained the large majority of the g- 6 glycan (Fig. 3D).

\section{Effect of Hyaluronidase on MAF}

Cesium chloride density gradients in the presence of $4 \mathrm{M} \mathrm{GuHCl}$ dissociated MAF into three fractions (Fernàndez-Busquets et al., 1996). The bottom of the gradient (F1) contained $5 \%$ of the total protein and carbohydrate. The middle fraction (F2) contained $90 \%$ of all protein and carbohydrate, while the upper fraction ( $F 3$ ) contained the remaining 5\%, mainly in the form of multiple protein bands. AFM images of F2 showed that it consisted of MAF rings with some arms still attached (Fig. 4C). In agarose gels stained

FIG. 2. Dissociation of MAF upon EDTA treatment. (A) AFM image of native MAFs. The inset shows details of arms (arrows) and of thinner linear structures (arrowheads). (B) AFM image of the sizing column-excluded fraction from EDTA-dissociated MAFs. (C) Detail of the only two components of the excluded fraction: rings and long molecules (arrowheads) with a length identical to the circumference of the rings. The color-encoded vertical z-scale of all images corresponds to $3 \mathrm{~nm}$.
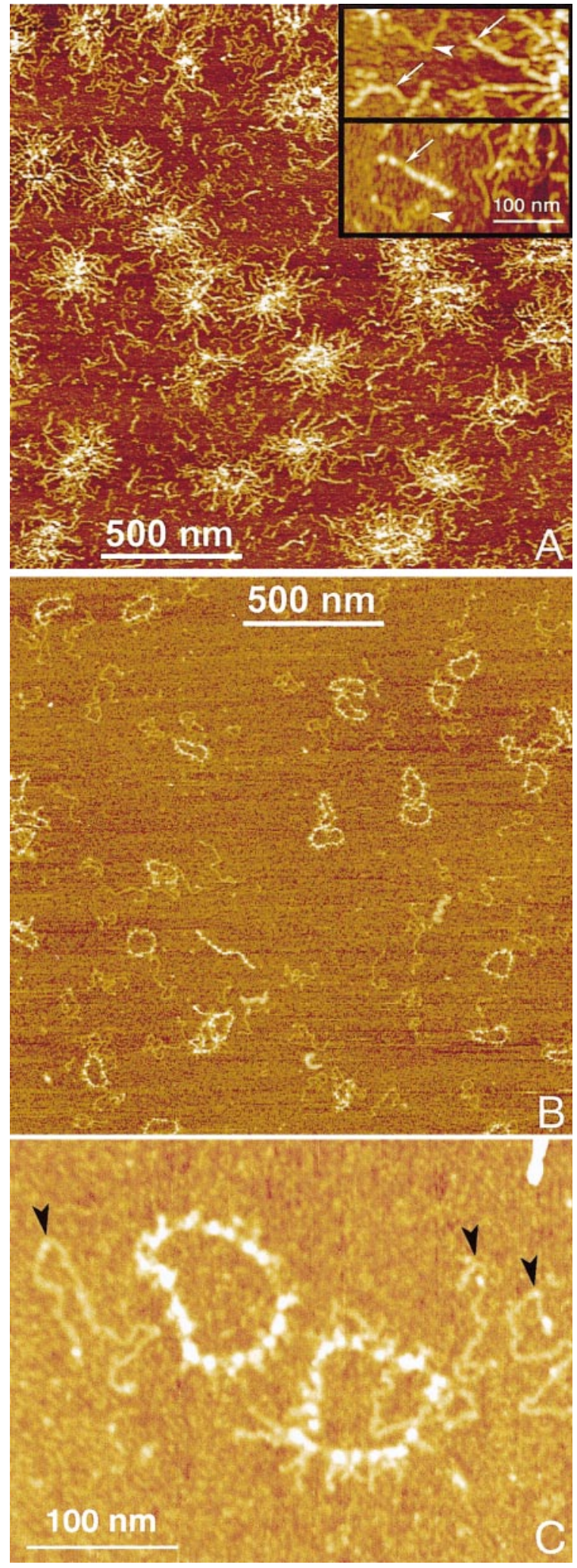


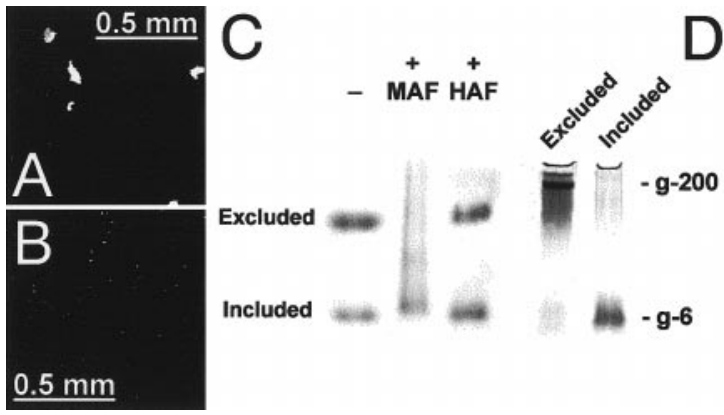

FIG. 3. Characterization of included and excluded fractions. ( $\mathrm{A}$ and $\mathrm{B}$ ) $\mathrm{Ca}^{2+}$-dependent aggregation assay at $20 \mathrm{mM} \mathrm{Ca}{ }^{2+}$ of synthetic beads coupled to excluded (A) and included (B) fractions. Confocal microscope pictures were taken after $3 \mathrm{~h}$ of aggregation. (C) Selective retention of the excluded fraction from EDTA-dissociated MAF by intact MAF but not by intact HAF . 100 $\mu \mathrm{g}$ of MAF (+MAF) or HAF (+HAF) was mixed with $20 \mu \mathrm{g}$ of EDTA-dissociated MAF and electrophoresed in a $1 \%$ agarose Tris-Tricine gel containing $15 \mathrm{mM} \mathrm{CaCl}_{2}$. Gels were subsequently stained with Alcian blue. Lane - , only EDTA-dissociated MAF loaded. (D) Glycans from the included and excluded fractions were prepared by pronase digestion and then separated by TBE-PAGE. Gels were stained with Alcian blue.

with Alcian blue, the incomplete dissociation in $\mathrm{GuHCl}$ was seen as a lower electrophoretic mobility of the arm-ring complex (Fig. 4E, lanes 3 and 5), compared to that of the naked ring obtained after EDTA dissociation (Fig. 4E, lane 7). Native MAF barely enters the gel (Fig. 4E, lane 1). Reducing conditions did not affect the mobility of any of the bands. After digestion of different F2 preparations and the MAF ring with hyaluronidase (HAse) from $\mathrm{S}$. hyalurolyticus, specific for hyaluronan (HA), the resulting substructures all had identical mobility (Fig. 4E, lanes 4, 6, and 8). The mobility of the ring from EDTA-dissociated MAF increased only slightly by this treatment (Fig. 4E, lanes 7 and 8). HAse digestion of native MAF (Fig. 4E, lane 2 ) was not as complete as that of dissociated MAF. The differences seen in the electrophoretic mobility of different F2 preparations are likely caused by the presence of a variable number of arms still remaining on the ring.

According to AFM visualization, HAse treatment leaves the ring intact, but completely eliminates the rod-like molecules also found in the excluded fraction. The susceptibility of the rod-like structures to treatment with the enzyme preparation suggested that they might be hyaluronan. This would be consistent with their AFM appearance; in particular, their 0.6-nm height is characteristic of single HA strands (Cowman et al., 1998). The presence or absence of HA was tested directly with FACE analyses (Calabro et al., 2000). Several MAF preparations were treated with a combination of streptococcal
HAse and chondroitinase ABC either directly or after known amounts of exogenous hyaluronan and chondroitin sulfate were added. The digests were fluorotagged and the products displayed by electrophoresis on polyacrylamide gels (data not shown). The disaccharides derived from exogenously added hyaluronan and chondroitin sulfate could be detected in samples containing MAF, indicating that the MAF preparation did not contain anything that
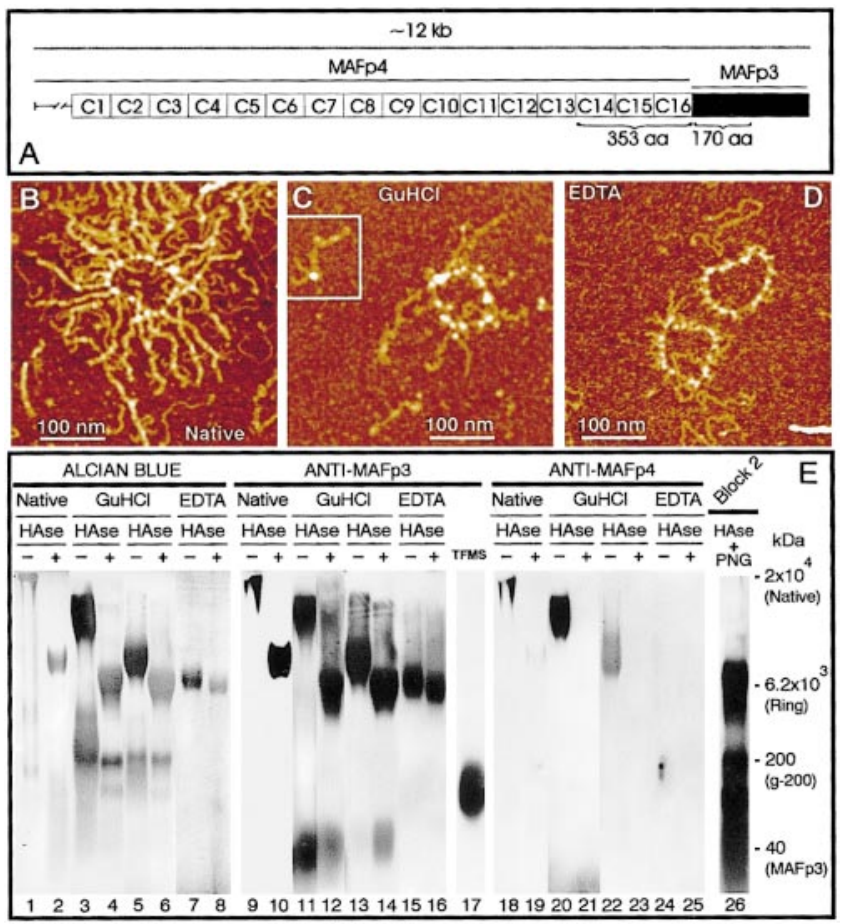

FIG. 4. Effect of hyaluronidase on MAF. (A) Scheme indicating the stretches of 353 and 170 residues used to raise polyclonal antibodies against MAFp4 and MAFp3, respectively. (B) AFM image of native MAF. (C) AFM image of the middle fraction (F2) of $\mathrm{GuHCl}$-dissociated MAF. The inset shows an isolated subunit containing one arm attached to one beaded structure from the ring from which two short chains protrude. (D) AFM image of the excluded fraction of EDTA-dissociated MAF. The color-encoded vertical z-scale of all AFM images corresponds to $3 \mathrm{~nm}$. (E) Native MAF (lane 1), two different F2 fractions (lanes 3 and 5), and the excluded fraction of EDTA-dissociated MAF (lane 7) were analyzed in a $1 \%$ agarose gel, transferred to a positively charged nylon membrane, and decorated with antibodies raised against MAFp3 (lanes 9-17), against MAFp4 (lanes 18-25), or against the g-200 glycan (lane 26) prior to staining with Alcian blue (lanes 1-8). $\sim 30 \mu \mathrm{g}$ of protein was loaded per lane. All fractions are shown before and after hyaluronidase digestion (-HAse and + HAse, respectively). The sample loaded in lane 3 is also shown after TFMS deglycosylation (lane 17) and after HAse+PNGase F treatment (lane 26). The mol ecular masses given are approximate and were deduced from a combination of SDS-PAGE analyses of the same samples (for the lower molecular masses) and from the addition of the masses of the different protein and carbohydrate components that are assembled in the larger structures. 
TABLE I

Molecular Dimensions of AFs from Three Different Sponge Species as Estimated from AFM I mages

\begin{tabular}{lcccc}
\hline \multicolumn{1}{c}{ AF } & Backbone shape & Backbone length $(\mathrm{nm})$ & Arm length $(\mathrm{nm})$ & Number of arms \\
\hline Microciona prolifera & Ring & 285 & 143 & $\sim 20$ \\
Halichondria panicea & Rod & 280 & 140 & $\sim 20$ \\
Ficulina ficus & Rod & 220 & 80 & $\sim 20$ \\
\hline
\end{tabular}

could inhibit the activities of the enzymes. No detectable bands were observed in the analysis of the MAF sample alone, indicating that hyaluronan and chondroitin sulfate, if present, are below detectable levels for the assay. In this case, the MAF sample contained $\sim 25 \mu \mathrm{g}$ of carbohydrate, and the conservative detection level of the analysis would have demonstrated a distinct disaccharide band if the original sample contained as little as $25 \mathrm{ng}$. FACE analyses of six additional samples of native MAF, F2, and the excluded fraction of EDTA-dissociated MAF also failed to detect hyaluronan products.

\section{Localization of Protein and Glycan Components in MAF}

Using rabbit polyclonal antibodies raised against MAFp3, we demonstrated that MAFp3 is found in the ring structure (Fig. 4E, lanes 9-16). Although most of MAFp3 is in the rings, a small pool exists as free forms (Fig. 4E, lanes 11-14). TFMS treatment of fraction $\mathrm{F} 2$ to remove carbohydrates disassembled the ring structure, converting it to what is probably monomeric MAF p3 (Fig. 4E, lane 17). The predominantly acidic composition of MAFp3 (pl 4.1) confers enough negative charge to this relatively small protein to allow its analysis in our $0.5 \times$ TBE agarose gels in the absence of SDS. The reduced electrophoretic mobility of deglycosylated MAFp3 in these agarose gels without SDS, then, is most likely the consequence of a decrease in the negative charge of the molecule after removal of acidic glycans.

The use of the monocl onal antibody Block 2, raised against a carbohydrate epitope present in g-200 but not in g-6 (Misevic and Burger, 1993), revealed that g-200 is present on the ring of MAF, on monomeric MAFp3, and also in a fraction strongly stained by Alcian blue in which MAFp3 and MAFp4 are absent (Fig. 4E, lane 26). This fraction has an apparent molecular mass of $\sim 200 \mathrm{kDa}$ according to control SDS-PAGE (data not shown), thus suggesting that it might represent free g-200. The signal obtained with Block 2 remains unchanged after PNGase F treatment, in agreement with previous data indicating that the $\mathrm{N}$-linkage of $\mathrm{g}-200$ is not sensitive to PNGase F digestion (Misevic and Burger, 1993).
Rabbit polyclonal antibodies raised against MAF p4 strongly recognized the ring structure in the $\mathrm{GuHCl}$-dissociated fractions of less electrophoretic mobility (Fig. 4E, lane 20). However, the higher the mobility, the less MAFp4 colocalizes with the ring (Fig. 4E, lanes 20, 22, and 24). Treatment with the Streptomyces hyaluronidase enzyme preparation invariably resulted in the total elimination of MAF p4 from the ring in any fraction (Fig. 4E, lanes 21, 23, and 25). Since MAFp4 is a large protein of $\sim 400 \mathrm{kDa}$ (Fernàndez-Busquets et al., 1998), it might be that its negative charge ( $\mathrm{pl} 3.2$ ) does not suffice either to make it enter our agarose gels in the absence of SDS or to transfer it to the nylon membrane. To investigate this possibility, we did SDS-PAGE analyses in 3-20\% gradient gels (Fig. 5).

SDS-PAGE followed by immunostaining with the polyclonal antibodies raised against MAFp3 revealed its presence in all fractions of the dissociative density gradient (Fig. 5, lanes 1-3). However, F2
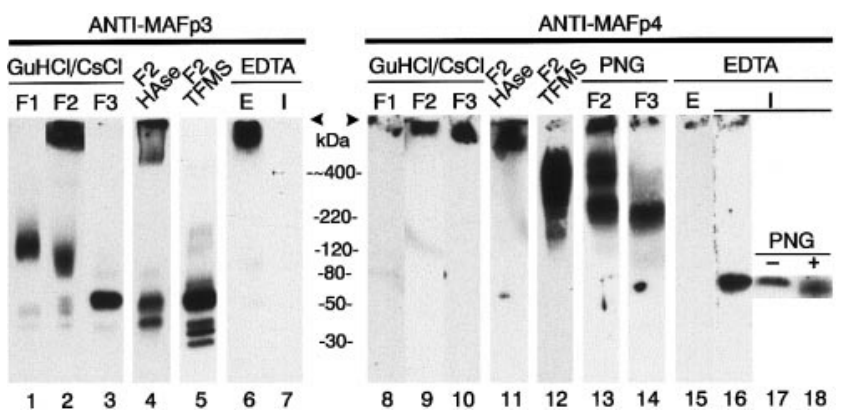

FIG. 5. The ring of MAF contains MAFp3 but not MAFp4. Different fractions obtained after dissociation and fractionation of MAF were loaded on a 3-20\% SDS-PAGE, electroblotted, and decorated with polyclonal antibodies raised against MAFp3 (lanes 1-7) or MAFp4 (lanes 8-18). GuHCl-dissociated MAF fractions F1, F2, and $F 3$ are shown. From EDTA-dissociated MAF, the excluded (E) and included (I) fractions are shown. F2 is also shown after HAse treatment (lanes 4 and 11), after TFMS deglycosylation (lanes 5 and 12), and after PNGase F digestion (lane 13). F 3 is also shown after PNGase F digestion (lane 14). The included fraction from EDTAdissociated MAF is shown again before and after PNGase F digestion (lanes 17 and 18, respectively). Arrowheads indicate the gel origin. The approximate amount of protein loaded per lane is 1.5 (F1, F3), 30 (F2), and $12 \mu \mathrm{g}(\mathrm{E}, \mathrm{I})$. 
was the only fraction where MAFp3 was detected in a component of very low electrophoretic mobility. AFM images revealed that F2 is the only fraction from the $\mathrm{GuHCl}-\mathrm{CsCl}$ gradients where rings are observed (Fig. 4C). After treatment with the Streptomyces HAse (Fig. 5, lane 4), the electrophoretic mobility of MAFp3 increased from 120 to $\sim 50 \mathrm{kDa}$, a molecular mass consistent with the deduced size from MAFp3 cDNA (Fernàndez-Busquets et al., 1998). TFMS-deglycosylated F2 (Fig. 5, lane 5) released significantly more MAFp3 than the hyaluronidase-treated F2. In EDTA-dissociated MAF all the MAFp3 signal was detected in the excluded fraction containing the ring (Fig. 5, lane 6). The use of polyclonal antibodies showed the presence of MAFp4 in F2 and F3 (Fig. 5, lanes 9 and 10). In SDS-polyacrylamide gels non-deglycosylated MAF p4 penetrated the $3 \%$ section after HAse digestion (Fig. 5, lane 11). TFMS treatment of F2 re vealed a broad band centered at $400 \mathrm{kDa}$ (Fig. 5, lane 12) that probably represents the full-length deglycosylated MAF p4, as predicted from Northern blot results (Fernàndez-Busquets et al., 1998). PNGase $F$ treatment releases the small g- 6 glycan from MAF (Misevic and Burger, 1990). PNGase $F$ digestion of $F 2$ and $F 3$ shifts the mobility of MAFp4 to that observed after TFMS deglycosylation. In PNGase F-treated F2 there is a strong signal at $\sim 400$ kDa (Fig. 5, lane 13), while in PN Gase F-treated F3 all the MAFp4 signal is in an $\sim 220-k D a$ band (Fig. 5, lane 14). This result correlates with our AFM observations that revealed that the arm fragments were longer in F 2 than in F3 (data not shown). In EDTA-dissociated MAF, all the MAFp4 signal was detected in the included fraction (Fig. 5, lane 16) as an $\sim 60-k D a$ structure. This is in accordance with our AFM observations of the included fraction that revealed an absence of large arm-like structures and suggested the existence of degradation processes affecting the arms. PNGase $F$ treatment slightly increased the electrophoretic mobility of the $\sim 60-\mathrm{kDa}$ band (Fig. 5, lanes 17 and 18), thus suggesting that it contained some g- 6 glycan or fragments of it.

As was already described for EDTA-dissociated MAF (Humphreys et al., 1977), GuHCl dissociation inactivates MAF irreversibly. We have tried to re constitute the original structure through stepwise dialysis of the disassembled components back to CaCMFTSW in the presence of protease inhibitors and at $4^{\circ} \mathrm{C}$. Although aggregated structures were formed, as assessed by polyacrylamide and agarose gel electrophoresis (data not shown), we failed to recover either the activity or the AFM morphology of both EDTA- and $\mathrm{GuHCl}$-dissociated factors.

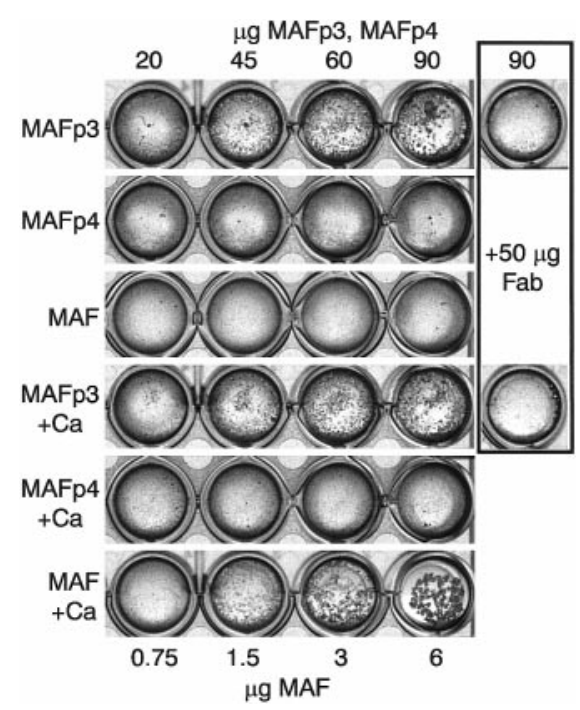

FIG. 6. Cell aggregation assay. Recombinant MAFp3, MAFp4, and native MAF were used in the absence of calcium at the protein concentrations indicated. Pictures were taken after 30 min of rotary shaking (top three rows). $\mathrm{Ca}^{2+}$ was then added to a final concentration of $10 \mathrm{mM}$, and stirring resumed for $30 \mathrm{~min}$, when a second set of pictures was taken (three bottom rows). As a control, $50 \mu \mathrm{g}$ of anti-MAF p3 Fab fragments was preincubated for $30 \mathrm{~min}$ with $90 \mu \mathrm{g}$ of recombinant MAFp3 before the assay was started (+ $50 \mu \mathrm{g} \mathrm{Fab).}$

\section{Cell Aggregation Activity of Recombinant MAFp3}

Recombinant MAF p3 was able to promote cell aggregation (Fig. 6), although at protein concentrations much higher than those of native MAF required to achieve a similar degree of aggregation. However, this effect appears to be specific, since (i) recombinant MAFp4 did not exhibit such activity at the same protein concentrations, (ii) preincubation of recombinant MAFp3 with Fab fragments raised against it prevented the formation of the cell aggre gates, and (iii) recombinant MAF p3-mediated aggregation was not observed with cells from other sponge species (data not shown). This cell aggregation activity of recombinant MAFp3 could also be observed in the absence of $\mathrm{Ca}^{2+}$.

\section{Aggregation Factors Bind a 68-kDa Lectin}

A protein of $68 \mathrm{kDa}$ was found to bind with high affinity to both MAF and M. prolifera cell surfaces (Varner, 1995). I n our cell membrane extracts from $\mathrm{M}$. prolifera and $\mathrm{H}$. panicea, a protein of $68 \mathrm{kDa}$ was detected in far-Western analyses by biotinylated AFs from several sponge species (Fig. 7A) and by glycans obtained from these AFs (Fig. 7B). The signal intensity resulting from AF binding to this protein varied from species to species, with extracts of M. prolifera giving the strongest signal with all AFs 
A

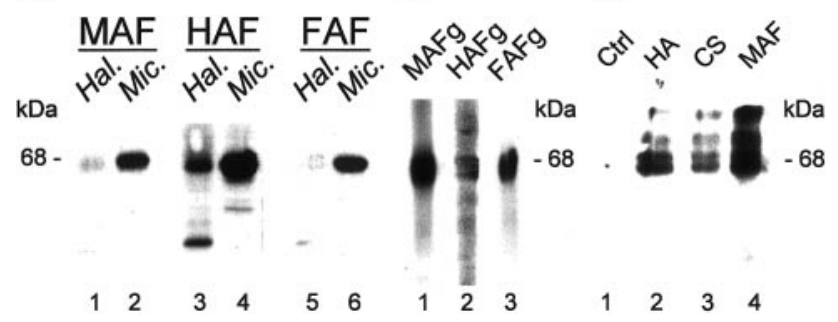

FIG. 7. Detection of a $68-k D a$ lectin in sponge cell membranes. (A) Aliquots of cell membrane extracts (10 $\mu \mathrm{g}$ of protein/ lane) from $\mathrm{H}$. panicea (lanes 1, 3, and 5) or M. prolifera (lanes 2, 4 , and 6) were electroblotted from a $12.5 \%$ SDS-PAGE. Blots were subsequently overlaid with biotinylated MAF in $2 \mathrm{mM}$ $\mathrm{CaCl}_{2}$ (lanes 1 and 2), biotinylated HAF (lanes 3 and 4), or biotinylated Ficulina AF (FAF, lanes 5 and 6) as indicated. (B) Cell membrane extracts from $M$. prolifera (10 $\mu \mathrm{g}$ of protein/lane) were loaded on a 7.5\% SDS-PAGE, blotted, and probed with biotinylated glycans from MAF (lane 1), HAF (lane 2), or FAF (lane 3) as indicated. (C) Hyaluronan (lane 2), chondroitin sulfate (lane 3), or MAF (lane 4) was coupled to EAH-Sepharose beads and subsequently incubated for $1 \mathrm{~h}$ with cell membrane extracts from M. prolifera (10 $\mu \mathrm{g}$ of protein). Bound material was released by boiling the bead pellet in $2 \%$ SDS, loaded on a $12.5 \%$ SDSPAGE, blotted, and detected using biotinylated MAF as described. Uncoupled EAH-Sepharose beads were used as a control (lane 1).

tested. A 68-kDa protein also bound to chondroitin sulfate-, MAF-, and hyaluronan-substituted EAH Sepharose beads (Fig. 7C).

\section{DISCUSSION}

If the circular backbone of MAF (Fig. 1B) were unfolded, the result would be a structure similar to the AF of $H$. panicea (Fig. IA), and one that resembles the classical structure of cartilage proteoglycan aggregates, with the arms of the AF corresponding to the proteoglycan monomer. Here we have shown that one of the two main proteins in MAF, MAFp3, is found exclusively in the ring, while the arms contain exclusively MAFp4 (Fig. 8A). As in most large proteoglycans (for a review, see Iozzo and Murdoch, 1996), the MAF p4 core protein has a modular structure made of tandem repeats (Fernàndez-Busquets et al., 1998). In our AFM images, the arms of MAF containing MAFp4 are about $140 \mathrm{~nm}$ long and have a beaded structure consisting of about 15 globules (Fig. 8B). This appearance has also been observed in proteoglycans like perlecan (Paulsson et al., 1987). However, MAF p4 does not have significant sequence homologies with any known proteoglycans. For the time being, its closest matches are found in two apparently unrelated proteins: the intracellular loop of $\mathrm{Na}^{+}-\mathrm{Ca}^{2+}$ exchangers (Fernàndez-Busquets et al., 1996) and a similar domain-structured endoglu- canase from the symbiotic bacterium Azorhizobium caulinodans (F ernàndez-Busquets et al., 1998); both share $30 \%$ identity with the MAFp4 repeats.

The small N-linked g-6 glycan (Misevic and Burger, 1990), involved in the binding of MAF to the cell receptors, is found in the included fraction of EDTA-dissociated MAF. This fraction exclusively contains MAFp4 but virtually no MAFp3, as shown by our Western blots decorated with the corresponding polyclonal antibodies. $\mathrm{g}-6$ is released from MAF upon PNGase F treatment (Misevic and Burger, 1990), and PNGase F digestion shifts the electrophoretic mobility of MAF p4 to its predicted deglycosylated size of $\sim 400 \mathrm{kDa}$ (Fig. 5). Therefore, g-6 is

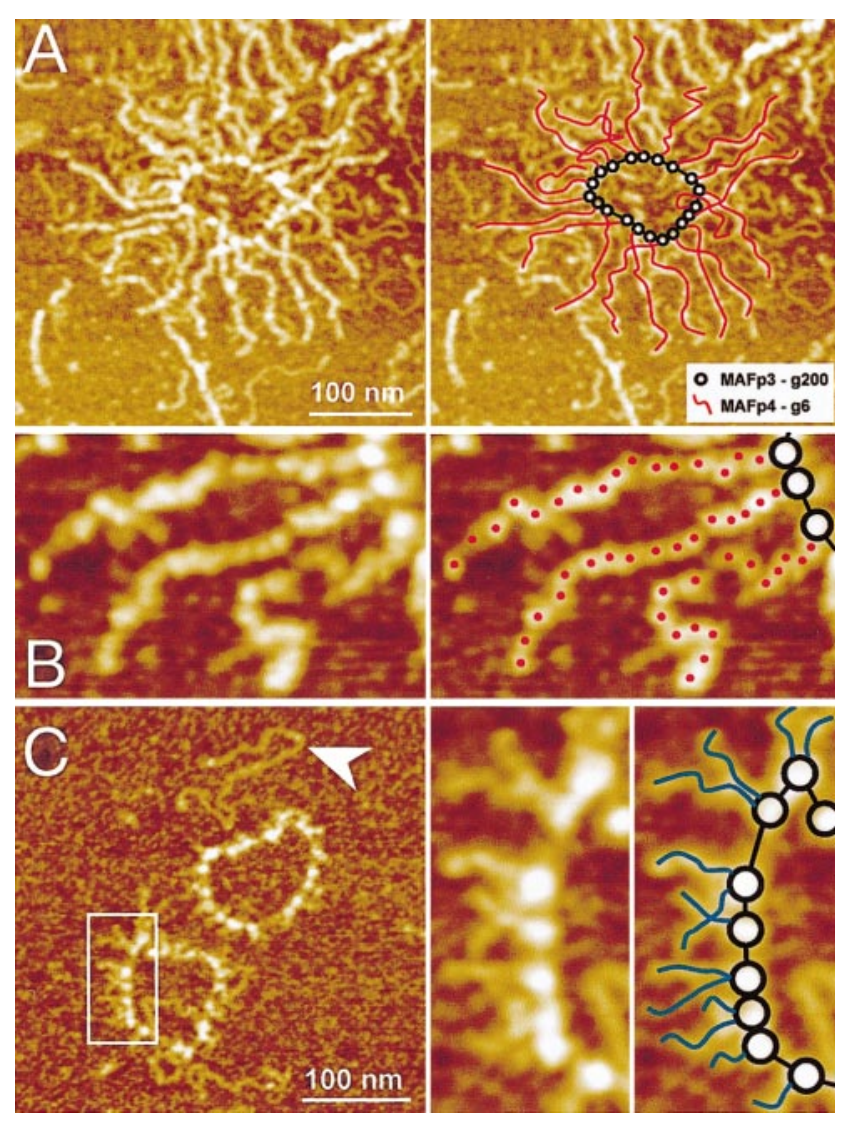

FIG. 8. Proposed model for the structure of MAF. (A) AFM image of native MAF showing the localization of MAFp3 in the ring (black circumferences) and of MAF p4 in the arms (red lines). MAFp3 carries the $g-200$ glycan and MAFp4 the $g-6$ glycan. (B) Detail of an AFM image of MAF showing the $\sim 15-16$ domains (red dots) observed in each arm in the native structure. (C) AFM image of MAF rings and of an isolated rod-like chain (arrowhead). We suggest that in the native AF the rod-like molecule runs along the circumference of the ring, stabilizing its interaction with the arms. The enlarged inset shows a detail of the ring structure with short chains protruding that might represent the g-200 glycan (blue lines). The color-encoded vertical z-scale of all the images corresponds to $3 \mathrm{~nm}$. 
the main glycan present on MAFp4. It was calculated that one MAF molecule has about 950 g-6 units (Misevic and Burger, 1990). That means that each of the 20 MAF arms will contain about 50 g- 6 units. The available cDNA-deduced sequence corresponding to about half of MAFp4 contains 40 potential $\mathrm{N}$-glycosylation sites (Fernàndez-Busquets et al., 1998), thus suggesting that MAF p4 can indeed contain all the g- 6 glycan present in MAF. The large $\mathrm{N}$-linked g-200 glycan, involved in the self-association of MAF (Misevic and Burger, 1993), is found in the excluded fraction of the EDTA-dissociated MAF. This fraction is composed of rings containing MAF p3 with virtually no MAF p4. g-200 is not released upon PNGase $F$ treatment of MAF (Misevic and Burger, 1993), and PNGase $F$ digestion of the MAF ring did not affect its electrophoretic mobility or its recognition by the monoclonal antibody Block 2 (Fig. 4), raised against a sulfated carbohydrate epitope (Spillmann et al., 1995) present in g-200 but not in g-6 (Misevic and Burger, 1993). Therefore, g-200 is the main glycan present on MAFp3. It was calculated that one MAF has $\sim 26 \mathrm{~g}-200$ units (Misevic and Burger, 1993). If each of the 20 globular structures observed in the MAF ring represents a MAFp3 molecule, we would expect one or two g-200 units on each MAFp3. Accordingly, the cDNA-deduced sequence of MAFp3 contains two to three putative $\mathrm{N}$-glycosylation sites, depending on the particular MAFp3 form (Fernàndez-Busquets et al., 1996, 1998; Fernàndez-Busquets and Burger, 1997). In our AFM images, one or two short linear structures protrude from each of the 20 globular structures forming the ring of MAF (Fig. 8C). Whenever MAF rings were observed to form dimers or larger aggregates (Fig. 2B), they appear to be interconnected through such short chains, thus suggesting that they are the g-200 glycan. In agreement with this hypothesis, their height $(0.55 \pm 0.1 \mathrm{~nm})$ is almost identical to the AFM thickness measurements of glycosaminoglycan chains like hyaluronan (Cowman et al., 1998).

Both g-6 and g-200 have a unique carbohydrate composition that includes glucuronic acid, fucose, mannose, galactose, $\mathrm{N}$-acetylglucosamine (Misevic and Burger, 1990, 1993), and a novel pyruvylated carbohydrate unit (Spillmann et al., 1993). g-6 has been shown to require a high degree of polyvalency for efficient cell binding (Misevic and Burger, 1990), and the small amount of it found in the ring fraction can therefore hardly account for this activity. Our results indicate that the ring structure is sufficient to mediate the $\mathrm{Ca}^{2+}$-dependent MAF self-association through g-200, while the arm structure would be involved in the $9-6$ binding to cell receptors.
The intact MAF has a $M_{r}$ of $\sim 2 \times 10^{7}$, and its protein content varies between 40 and $50 \%$ (Henkart et al., 1973). MAF p3 and MAFp4 are translated from a single mRNA of $\sim 12 \mathrm{~kb}$ (Fernàndez-Busquets et al., 1998), which would account for a total molecular mass of $\sim 440 \mathrm{kDa}$. Twenty MAFp3 + MAFp4 units, then, would represent $\sim 45 \%$ of the total mass of MAF, a figure in accordance with the hypothesis that MAFp3 and MAFp4 are the main protein components of MAF. Therefore, we propose that each of the 20 globular structures forming the MAF ring is a g-200-glycosylated MAF p3 unit, and each of the 20 arms is a g-6-glycosylated MAFp4 unit. As the ring is not sensitive to reducing conditions (Humphreys et al., 1977), its stabilization cannot be based on disulfide bonds between protein chains. Chemical deglycosylation, on the other hand, completely disrupts the ring, suggesting that interactions between glycans or between glycans and protein might be responsible for its integrity, although such treatment can affect other protein-mediated interactions contributing to the stabilization of the ring structure. However, it is unlikely that the ring is stabilized by covalent peptide bonds, because neither MAFp3 nor MAFp4 is internally cleaved by our TFMS treatment, and both retain their predicted original masses of $\sim 50$ and $\sim 400 \mathrm{kDa}$, respectively. Each of the 20 arms is attached to one of the 20 globular structures in the ring in a 1:1 stoichiometry. $\mathrm{GuHCl}, \mathrm{HAse}$, and EDTA are able to remove the arms without affecting the ring, thus suggesting that although MAF p3 and MAF p4 are contiguous in their mRNA, they are not covalently connected in the final structure. The MAFp3-containing ring structure is rich in protein, and any significant protease activity would be expected to open it. Although both HAse and EDTA treatments might risk contaminating low-level protease activities, we have seldom observed linearized rings in our AFM images. Moreover, neither MAF p3 nor MAF p4 is internally cleaved after extended HAse digestion (see Fig. 5, lanes 2, 4, 9, and 11). In GuHCl dissociative density gradients, which do not cleave covalent bonds, fraction F3 contains both MAFp3 and MAFp4. Finally, SDS does not inhibit the dissociation of the arms from the ring in the presence of EDTA (Humphreys et al., 1977), suggesting that this dissociation is not due to hydrolytic enzymes. These results reinforce the hypothesis that MAFp3 and MAFp4 are two separate proteins in the native AF.

As an interesting analogy, cadherins undergo a large conformational change in the absence of $\mathrm{Ca}^{2+}$ and, as a result, are rapidly degraded by proteases, to which they are highly sensitive (Takeichi, 1988). Cells expressing distinct cadherins aggregate sepa- 
rately when mixed in culture (Geiger and Ayalon, 1992), a phenomenon that resembles the speciesspecific cell adhesion activity of sponge AFs. Multiple putative $\mathrm{Ca}^{2+}$-binding motifs of the types DXD and DXNDN, characteristic of cadherins (Pigott and Power, 1993), are present in MAFp4 (FernàndezBusquets et al., 1998).

All previous evidence has indicated that the selfassociation of MAF involves $\mathrm{Ca}^{2+}$-dependent interactions of its carbohydrate moieties (Misevic and Burger, 1993). Here we have shown that glycan-free recombinant MAF p3 promotes specific cell aggregation of Microciona cells in the absence of $\mathrm{Ca}^{2+}$, suggesting a direct rolefor the protein moiety of MAF in sponge cell adhesion. This activity was never detected when working in vitro with native MAF, probably due either to a shielding of the core protein by the exposed glycans or to difficulties in using the high concentrations of native MAF needed to achieve the required amount of MAFp3. This situation seems to be different in vivo. According to J umblatt et al. (1980), while as few as 400 molecules of AF bound per cell are sufficient to promote aggregation in the standard assay, the actual amount in vivo was calculated to be about 28000 molecules per cell. This might provide the high concentration of MAFp3 observed to promote in vitro aggregation. From MAF cell-binding studies, species-specificity of AF binding to cells had been proposed previously (J umblatt et al., 1980). Our results suggest that different sponge species might carry different amounts of receptor protein on their surface (Fig. 7A) and that this quantitative component could also contribute to determining specificity.

A key component of classical proteoglycan aggregation is hyaluronan. Using peptides derived from a putative HA-binding domain found in MAFp3 (Fernàndez-Busquets et al., 1996), Kuhns et al. (1998) have suggested a direct interaction between $\mathrm{HA}$ and MAF p3. The HAse treatment protocol used in our study completely abolishes the cell aggregation activity of MAF (data not shown), a result in accordance with our previous observation that addition of exogenous HA to MAF preparations with low activity raised the aggregation efficiency of the factor to that found in the most active preparations (Fernàndez-Busquets et al., 1996). Here, we have presented clear shifts in the electrophoretic mobility of the MAF ring and of MAFp3 and MAFp4 upon S. hyalurolyticus HAse treatment, with concurrent elimination of the rod-like molecules with lengths equal to the ring circumferences. These data suggested that the rod-like structures, with width characteristic of a polysaccharide, might be HA. However, we did not detect any hyaluronan-derived oligosaccha- rides in FACE assays. Since sponges are noteworthy for their singular glycan structures (Spillmann et al., 1993, 1995), we now suggest that our HAse treatment cleaves bonds between $\mathrm{N}$-acetylglucosamine and glucuronic acid in select subregions of a polysaccharide. Alternatively, a contaminating glycosidase may cleave susceptible bonds in the structure. The susceptible sites are probably nonrepetitive, since we have failed to detect defined small oligosaccharides in the FACE analyses.

Sponges were the first animals faced with multicellularity, and all their cells are extraordinarily motile. The fact that AFs mediate sponge cell adhesion suggests that proteoglycan-like macromolecules were one of the first extracellular matrix molecules to appear and that their structure was originally designed to allow a precise modulation of their adhesive properties. MAFp3 and MAFp4 do not share any sequence homology with hyaladherins, molecules that interact with hyaluronan, or with any known proteoglycan core protein. Therefore, sponge aggregation factors are the first described members of a new family of macromolecules that have characteristics of proteoglycans and that have a central role in the specificity of cell adhesion.

We thank Andrew P. Spicer for helpful discussions, and Ruth Chiquet, J an Hofsteenge, and Dorothe Spillmann for critical comments on the manuscript.

\section{REFERENCES}

Björnsson, S. (1993) Size-dependent separation of proteoglycans by electrophoresis in gels of pure agarose, Anal. Biochem. 210, 292-298.

Calabro, A., Benavides, M., Tammi, M., Hascall, V. C., and Midura, R. J . (2000) Microanalysis of enzyme digests of hyaluronan and chondroitin/dermatan sulfate by fluorophore-assisted carbohydrate electrophoresis (FACE), Glycobiology 10, 273-281.

Cowman, M. K., Li, M., and Balazs, E. A. (1998) Tapping mode atomic force microscopy of hyaluronan: Extended and intramolecularly interacting chains, Biophys. J . 75, 2030-2037.

Edelman, G. M., and Crossin, K. L. (1991) Cell adhesion molecules: Implications for a molecular histology, Annu. Rev. Biochem. 60, 155-190.

Edge, A. S. B., Faltynek, C. R., Hof, L., Reichert, L. E., J r., and Weber, P. (1981) Deglycosylation of glycoproteins by trifluoromethanesulfonic acid, Anal. Biochem. 118, 131-137.

Fedarko, N. S. (1993) I solation and purification of proteoglycans, Experientia 49, 369-373.

Fernàndez-Busquets, X., and Burger, M. M. (1997) The main protein of the aggregation factor responsible for species-specific cell adhesion in the marine sponge Microciona prolifera is highly polymorphic, J. Biol. Chem. 272, 27839-27847.

Fernàndez-Busquets, X., and Burger, M. M. (1999) Cell adhesion and histocompatibility in sponges, Microsc. Res. Tech. 44, 204218.

Fernàndez-Busquets, X., Gerosa, D., Hess, D., and Burger, M. M. (1998) Accumulation in marine sponge grafts of the mRNA 
encoding the main proteins of the cell adhesion system, J . Biol. Chem. 273, 29545-29553.

Fernàndez-Busquets, X., Kammerer, R. A., and Burger, M. M. (1996) A 35-kDa protein is the basic unit of the core from the $2 \times 10^{4}-k \mathrm{Da}$ aggregation factor responsible for species-specific cell adhesion in the marine sponge Microciona prolifera, J . Biol. Chem. 271, 23558-23565.

Finne, J ., and Krusius, T. (1982) Preparation and fractionation of glycopeptides, Methods Enzymol. 83, 269-277.

Fritz, J ., Anselmetti, D., J archow, J ., and Fernàndez-Busquets, X. (1997) Probing single biomolecules with atomic force microscopy, J . Struct. Biol. 119, 165-171.

Geiger, B., and Ayalon, O. (1992) Cadherins, Annu. Rev. Cell Biol . 8, 307-332.

Hardingham, T. E., and F osang, A. J . (1992) Proteoglycans: Many forms and many functions, FASEB J . 6, 861- 870.

Harlow, E., and Lane, D. (1988) Antibodies: A Laboratory Manual, pp. 53-631, Cold Spring Harbor Laboratory Press, Cold Spring Harbor.

Henkart, P., Humphreys, S., and Humphreys, T. (1973) Characterization of sponge aggregation factor. A unique proteoglycan complex, Biochemistry 12, 3045-3050.

Humphreys, S., Humphreys, T., and Sano, J . (1977) Organization and polysaccharides of sponge aggregation factor, J . Supramol. Struct. 7, 339-351.

I ozzo, R. V. (1998) Matrix proteoglycans: From molecular design to cellular function, Annu. Rev. Biochem. 67, 609- 652.

Iozzo, R. V., and Murdoch, A. D. (1996) Proteoglycans of the extracellular environment: Clues from the gene and protein side offer novel perspectives in molecular diversity and function, FASEB J . 10, 598-614.

J archow, J ., and Burger, M. M. (1998) Species-specific association of the cell-aggregation molecule mediates recognition in marine sponges, Cell Adhes. Commun. 6, 405- 414.

J umblatt, J . E., Schlup, V., and Burger, M. M. (1980) Cell-cell recognition: Specific binding of Microciona sponge aggregation factor to homotypic cells and the role of calcium ions, Biochemistry 19, 1038-1042.

Kuhns, W. J ., Fernàndez-Busquets, X., Burger, M. M., Ho, M., and Turley, E. (1998) Hyaluronic acid-receptor binding demonstrated by synthetic adhesive proteoglycan peptide constructs and by cell receptors on the marine sponge Microciona prolifera, Biol. Bull. 195, 216-218.

Laemmli, U. K. (1970) Cleavage of structural proteins during the assembly of the head of bacteriophage T4, Nature 227, 680685.

Lyubchenko, Y., Shlyakhtenko, L., Harrington, R., Oden, P., and Lindsay, S. (1993) Atomic force microscopy of Iong DNA: I maging in air and under water, Proc. Natl. Acad. Sci. USA 90, 2137-2140.

Misevic, G. N. (1989) Immunoblotting and immunobinding of acidic polysaccharides separated by gel electrophoresis, Methods Enzymol. 179, 95-104.

Misevic, G. N., and Burger, M. M. (1990) The species-specific cell-binding site of the aggregation factor from the sponge $\mathrm{Mi}$ crociona prolifera is a highly repetitive novel glycan containing glucuronic acid, fucose, and mannose, J. Biol. Chem. 265, 20577-20584.
Misevic, G. N., and Burger, M. M. (1993) Carbohydrate-carbohydrate interactions of a novel acidic glycan can mediate sponge cell adhesion, J . Biol. Chem. 268, 4922- 4929.

Misevic, G. N., Finne, J ., and Burger, M. M. (1987) Involvement of carbohydrates as multiple low affinity interaction sites in the self-association of the aggregation factor from the marine sponge Microciona prolifera, J . Biol. Chem. 262, 5870-5877.

Müller, W. E. G., Gamulin, V., Rinkevich, B., Spreitzer, I., Weinblum, D., and Schröder, H. C. (1994) U biquitin and ubiquitination in cells from the marine sponge Geodia cydonium, Biol. Chem. Hoppe Seyler 375, 53- 60.

Müller, W. E. G., Müller, I., and Zahn, R. K. (1976) Speciesspecific aggregation factor in sponges. V. Influence on programmed syntheses, Biochim. Biophys. Acta 418, 217-225.

Paulsson, M., Yurchenko, P. D., Ruben, G. C., Engel, J ., and Timpl, R. (1987) Structure of low density heparan sulfate proteoglycan isolated from a mouse tumor basement membrane, J . Mol. Biol. 197, 297-313.

Pfeifer, K., Frank, W., Schröder, H. C., Gamulin, V., Rinkevich, B., Batel, R., Müller, I . M., and Müller, W. E. G. (1993) Cloning of the polyubiquitin cDNA from the marine sponge Geodia cydonium and its preferential expression during reaggregation of cells, J . Cell Sci. 106, 545-553.

Pigott, R., and Power, C. (1993) The cadherin family, in The Adhesion Molecule Factsbook, 1st ed., pp. 6-8, Academic Press, London.

Rottmann, M., Schröder, H. C., Gramzow, M., Renneisen, K., Kurelec, B., Dorn, A., Friese, U., and Müller, W. E. G. (1987) Specific phosphorylation of proteins in pore complex-laminae from the sponge Geodia cydonium by the homologous aggregation factor and phorbol ester. Role of protein kinase $C$ in the phosphorylation of DNA topoisomerase II, EMBO J . 6, 39393944.

Sambrook, J ., Fritsch, E. F., and Maniatis, T. (1989) Molecular Cloning: A Laboratory Manual, 2nd ed., pp. 9.42-9.44, Cold Spring Harbor Laboratory Press, Cold Spring Harbor, NY.

Schröder, H. C., Kuchino, Y., Gramzow, M., Kurelec, B., Friese, U., Uhlenbruck, G., and Müller, W. E. G. (1988) Induction of ras gene expression by homologous aggregation factor in cells from the sponge Geodia cydonium, J . Biol. Chem. 263, 16334-16340.

Spillmann, D., Hård, K., Thomas-Oates, J ., Vliegenthart, J . F . G., Misevic, G., Burger, M. M., and Finne, J . (1993) Characterization of a novel pyruvylated carbohydrate unit implicated in the cell aggregation of the marine sponge Microciona prolifera, J . Biol. Chem. 268, 13378-13387.

Spillmann, D., Thomas-Oates, J . E., van Kuik, J . A., Vliegenthart, J . F. G., Misevic, G., Burger, M. M., and Finne, J . (1995) Characterization of a novel sulfated carbohydrate unit implicated in the carbohydrate-carbohydrate-mediated cell aggregation of the marine sponge Microciona prolifera, J. Biol. Chem. 270, 5089-5097.

Takeichi, M. (1988) The cadherins: Cell-cell adhesion molecules controlling animal morphogenesis, Development 102, 639- 655.

Varner, J . A. (1995) Cell adhesion in sponges: Potentiation by a cell surface $68 \mathrm{kDa}$ proteoglycan-binding protein, J. Cell Sci. 108, 3119-3126.

Weinbaum, G., and Burger, M. M. (1973) Two component system for surface guided reassociation of animal cells, Nature 244, 510-512. 